\title{
TAHSP:-
}

The Internet Joưnal of Allied Health Sciences and Practice

A Peer Reviewed Publication of the College of Health Care Sciences at Nova Southeastern University

Dedicated to allied health professional practice and education

http://ijahsp.nova.edu Vol. 12 No. 3 ISSN 1540-580X

\section{Effect of Cueing on Learning Transfer Among Health Profession Students Engaged in a Case-based Analogical Reasoning Exercise}

\author{
Timothy Speicher, PhD, ATC, CSCS, PRT 1 \\ Alexandra Bell, PhD, $\mathrm{PT}^{2}$ \\ Marijke Kehrhahn, $\mathrm{PhD}^{3}$ \\ Douglas Casa, PhD, ATC, FACSM, FNATA4
}

1. Assistant Professor, DSc Health Sciences Program, Rocky Mountain University of Health Professions, Provo, UT

2. Associate Professor and Adult Program Section Head, University of Connecticut, Storrs, CT

3. Associate Professor of Adult Learning \& Associate Dean, University of Connecticut, Storrs, CT

4. Professor \& Director of the Athletic Training Education, University of Connecticut, Storrs, CT

United States

CITATION: Speicher T, Bell A, Kehrhahn M, Casa D. Effect of Cueing on Learning Transfer Among Health Professions Students Engaged in a Case-based Analogical Reasoning Exercise. The Internet Journal of Allied Health Sciences and Practice. July 2014. Volume 12 Number 3.

\begin{abstract}
Purpose: The purpose of this study was to examine the effect of cueing on learning transfer among pre-professional, health profession undergraduates engaged in a case-based analogical reasoning exercise. Methods: The study design was a quasiexperimental, randomized post-test design. Volunteer undergraduate pre-professional health profession students $(N=192)$ were assigned to either an experimental group, which received two written clinical cases with cues or the control, which received the same cases, but no cues. After participants read the cases, a target case was provided for solution based on the previous cases. Target case solutions were scored by content experts for extent of transfer of a structural principle and optimal treatment method implicitly embedded in the comparison cases. Results: Cued participants $(n=98)$ demonstrated significantly more learning transfer $(t(175.91)=2.65 ; p=.009 ; d=.39)$ of the structural principle than non-cued participants $(n=94)$. However, no significant difference was found for cued and non-cued groups for transfer of the optimal treatment method $(t(190)=.874 ; p=$ .39; and a small effect size $(d=.13)$ was present. Conclusion: Learning transfer improves among pre-professional health profession undergraduates when cued during a case-based analogical reasoning experience. The findings support and extend previous multiple case-based analogical reasoning study outcomes indicating that multiple patient cases with cueing should be utilized over single case examination with or without cueing to promote transfer of learning. Improvements in learning transfer may lead to improved quality of patient care.
\end{abstract}

\section{INTRODUCTION}

Transfer of learning has been characterized as the ability to use knowledge or skill obtained in one context to solve a problem in another context. ${ }^{1}$ Near transfer occurs when the two contexts are similar, and far transfer when the two contexts are dissimilar. In allied health education, instructors expect students will acquire the ability to transfer their classroom learning of patient cases to solve novel clinical problems in practice.2,3 One of the most common methods employed by classroom educators to promote students' transfer and problem-solving is examination of a single patient case, a pedagogical practice shown to be relatively ineffective in promoting transfer. ${ }^{3-5}$ However, researchers have demonstrated that using multiple patient cases with instructor cueing (i.e., prompting or providing hints about salient aspects of the cases) can be a very effective instructional strategy for fostering transfer of learning to enable novel problem solving $4 ., 5$ The value of using multiple case comparisons was demonstrated 
among health sciences undergraduates $(N=35)$ in a study by Norman et al. who found a very large effect size difference $(d=$ 1.36) in solving clinical case problems between students who examined multiple case examples and those who utilized one example. ${ }^{5}$

Multiple case comparison supports students' analogical reasoning abilities. ${ }^{5}$ Analogical reasoning involves the ability to perceive and use relational similarity between two situations, events, or patient cases. ${ }^{6,7}$ In order to compare two situations or events, a familiar domain of knowledge known as a base or source is utilized to comprehend an unfamiliar domain known as a target. ${ }^{6}$ By appreciating commonalities between the base and target, individuals can make inferences of relationship between them and generate a shared structural principle about that relationship. ${ }^{6}$ According to Gentner's structure-mapping theory, a structural principle is a set of correspondences between cases or experiences. ${ }^{8}$ For example, students might be presented two patient cases with one involving heat exhaustion and the other heat stroke. Through provision of either written or verbal cues from the instructor, students are prompted to compare the cases to uncover an implicit underlying principle shared by both cases. Once discovered, the structural principle can be used to either diagnose or treat a patient who would benefit from the application of the principle. In this example, the structural principle causing the symptoms may be the lack of thermoregulation of core body temperature. When cueing (i.e., providing hints to encourage comparison) is coupled with the analogical reasoning process, transfer of the structural principle to a target problem or clinical case is enhanced.4,5,9

The value of multiple case comparisons in supporting transfer of learning can be enhanced when instructors provide cues or prompts to students about what features of the cases are most salient in transferring to new problems. Holyoak and Koh asserted that providing learners with cueing is a critical instructional technique for moving students to identify structural relationships among multiple analogues or examples because it alerts them to look for relationships that exist among analogues for solving novel problems. ${ }^{10}$ The study by Norman et al described earlier in this paper demonstrated not only that multiple case analysis was superior to single case analysis, but also that students who utilized multiple cases and received cues regarding how cases were analogous outperformed students who just utilized multiple cases $(d=1.36)$ alone. ${ }^{5}$

Outcomes of studies by Norman et al and Shayo and Olfman highlight the central role instructors serve in the case-based analogical reasoning process. ${ }^{5,11}$ Instructors can cue learners to identify structural relationships that exist between cases being compared and learners' past experiences. Speicher and Kehrhahn explain that the cueing intervention helps learners retrieve and map their present learning experience with their past experience. ${ }^{12}$ The mapping process helps to build not only an individual's schema, but the match between both develops in the student a perception of being able to apply the learning experience to a current or future problem. ${ }^{12}$

Learners' ability to recognize underlying structural similarities across cases and to apply underlying principles to solve novel problems is influenced by their level of experience and expertise in the domain. ${ }^{13}$ Individuals with more experience have more complex cognitive schema representing their knowledge and contextual aspects of their experience in a domain. ${ }^{14}$ Subsequently, they are able to identify structural relationships and rules for problem solving. ${ }^{15}$ In contrast, novices have been shown to rely more on surface characteristics of an experience (e.g., skin color) rather than identifying and utilizing a structural principle (e.g., thermoregulation of core body temperature) for problem solving when they lack experience in a particular domain. ${ }^{13}$

In allied health education programs, students in the pre-professional coursework phase are just beginning to develop their domain-specific knowledge. As novices, they have a limited experiential base and less complex schema from which to draw analogies and apply to problem solving. The purpose of this study was to expand on the work of Norman et al by examining the effect of cueing on learning transfer among pre-professional, health profession undergraduates engaged in a multiple casebased analogical reasoning exercise. The findings of this study have implications for how health profession instructors can utilize patient case examples to foster transfer and clinical decision making skills. Improvements in learning transfer may lead to improved quality of patient care.

\section{METHODS}

A quasi-experimental, randomized post-test design was utilized. ${ }^{16}$ The sample consisted of 192 volunteer pre-professional undergraduate students seeking a career in allied health or medicine. Students were college-aged $(M=19$ years, $S D \pm 1.73)$, from the Northeast (92\%), and primarily Caucasian (87\%). Participants were randomized into either experimental $(n=98 ; 40$ males; 58 females) or control ( $n=94 ; 43$ males; 51 females) conditions. The study occurred at four institutions of higher education in the State of Connecticut with Institutional Review Board approval.

The experimental and control groups compared two worked cases (i.e., cases with solutions) involving patients who had experienced heat illness (Figure 1). The experimental group (cued) received written cues guiding participants to look for a 
solution common to both cases and to write down how each case was similar. The instruction for the control group (non-cued) was to read and write down what was going on in each case separately. Cueing prompts and their presentation format were based on the work of Gentner et al and Gick and Holyoak.4,17 Specifically, cues were designed to move the participant to compare both cases. Additionally, based on procedures used by previous researchers to facilitate case comparison, patient cases for the experimental group appeared on the same sheet of paper with cases for the control group on separate sheets. ${ }^{4}$ Imbedded in the worked cases was an implicit shared structural principle (thermoregulation of core body temperature) applied through an optimal treatment method (direct full body technique). After examining the cases, members of each group composed a treatment solution for a third target case involving a hypothermic patient (Figure 2).

Figure 1. Written Cases with Cues

Please read the following two cases. Comparison of the two cases will help you produce a solution(s) for a third test case.

Case 1:

You are the clinician responsible for a youth soccer camp. A camper approaches you after lunch and complains she does not feel well. She tells you she feels sick to her stomach, is tired, and has a headache.

You notice her breathing is elevated, skin is pale and she is sweating profusely. She is having difficulty standing so you ask her to sit down. You take her temperature and it is 103 degrees Fahrenheit. You move the camper to a cool environment and start your treatment, which consists of placing towels dipped in cold water over her body. You retake her temperature a few minutes after the treatment and it has dropped to 102 degrees Fahrenheit, but the breathing rate and skin color are the same as before.

Case 2:

You are a health care provider at a local road race. As a runner is approaching the finish line, he stumbles and collapses.

When you approach the runner, his face is red. There is no sweat on his body and his breathing is very rapid. You ask him questions, but cannot understand them because his speech is slurred. You move the runner to the medical tent and take his rectal temperature, which is 106 degrees Fahrenheit. You place him in a kiddy pool of cool water. You retake his temperature a few minutes after the treatment and it has dropped to 102 degrees Fahrenheit, the breathing rate has slowed, the face redness has diminished and he is now able to speak a few coherent words.

Think about the similarities between these two cases. What are the key similarities in the two cases? Write them down below. 
Figure 2. Target Case

\begin{tabular}{|l|}
$\begin{array}{l}\text { Provide a solution(s) for this case based on what you have learned thus far from the cases } \\
\text { examined. }\end{array}$ \\
$\begin{array}{l}\text { You enter the hydrotherapy room in the therapy clinic to care for a patient who has "passed out" } \\
\text { while receiving a full-body cold whirlpool treatment. Several individuals are assistant him out of } \\
\text { the pool, but he appears to be unable to exert any coordinated physical effort to get out even with } \\
\text { assistance. You notice the temperature of the whirlpool is } 45 \text { degrees Fahrenheit. Upon removing } \\
\text { the individual from the pool, you notice his skin is pale, lips are slightly blue, his breathing is } \\
\text { extremely slow, but he is shivering. You ask him questions, but cannot understand him because } \\
\text { his speech is slurred. }\end{array}$ \\
\hline What should you do and why? Be specific as you can.
\end{tabular}

The dependent variables of the study were 1) transfer of the structural principle, and 2) transfer of the optimal treatment method common to the worked cases to the solution for the target case. The independent variable was the cueing intervention (cued or not cued). Covariates included in the analyses were prior experience with case content, and because the cases involved environmental conditions, geographic location where subjects spent most of their lives.

A demographic and prior experience survey (DPES) (See appendix A) was administered. No significant differences were found within or between groups, revealing a sample homogenous in nature. Also, no significant differences in prior experience with case content between groups existed.

\section{Data Collection}

Guided by the work of Gentner and Colhoun, the authors developed a learning transfer assessment instrument (LTAl) (See appendix B) to determine the extent to which participants transferred the worked cases' structural principle and optimal treatment method to a third target case. ${ }^{18}$ The LTAI utilized an ordinal scale from 0 to 3 to determine the extent of structural similarity in participant solutions. A score of 3 indicated a great extent of structural similarity and 0 represented none. Two trained raters with expertise in core body temperature thermoregulation assessed participants' written solutions to the third case using the LTAI. Intraclass correlation coefficients (ICC) indicated that LTAI outcomes had high inter- and intra-rater reliability. Inter-rater reliability of the structural principle and treatment method were .91 and .95 , respectively. Intra-rater reliability ranged from .88 to .93 for the structural principle and from .95 to .96 for the treatment method.

Data collection occurred through the LTAI and DPES instruments in a one-time, 30-minute classroom environment. After worked cases were read and responses given, participants were provided the target case to be solved. In order to limit unintentional case comparison within the control group, participants were not permitted to refer back to their worked cases or responses for developing their solution of the third target case.

\section{Data Analysis}

Statistical analysis was performed with SPSS v. 16 with an alpha level of .05 (two-tailed) as the criterion for significance. An independent-samples $t$-test analysis of the mean difference was calculated to assess the extent of transfer of the structural principle and treatment method between groups. Additionally, effect size for mean differences $(d)$ were calculated according to Cohen's conventions. ${ }^{19}$ Correlational analysis was performed prior to ANCOVA testing to determine variance in transfer scores based on group assignment, level of prior experience, and geographic location.

\section{RESULTS}

Table 1 outlines the descriptive statistics for the study. The cued group scored significantly higher than the non-cued group for transfer of the structural principle $\left(t(175.91)=2.65 ; p=.009 ; \mathrm{Cl}_{95}=(.10,0.68)\right.$, and the size of the effect was medium-small $(d=$ .39). Equal variances were not assumed according to Levene's Test for equality of variances $(p=>.05)$. However, no significant difference was found in mean scores between cued and non-cued groups for transfer of the optimal treatment method $(t(190)=$ $.874 ; p=.39 ; \mathrm{Cl}_{95}=(-0.14,0.36)$, and a small effect size $(d=.13)$ was present. 
Table 1. Mean Difference of Transfer Scores

\begin{tabular}{llllll}
\hline Outcome Variable & Cued Participants & $\begin{array}{l}\text { Non-Cued } \\
\text { Participants }\end{array}$ & $t$ Value & $p$ Value & $\begin{array}{l}\text { Effect Size } \\
\text { (d) }\end{array}$ \\
\hline Structural Principle & $\mathrm{M}=2.30$ & $\mathrm{M}=2.14$ & $(175.91) 2.65$ & .009 & .39 \\
& $\mathrm{SD}=.89$ & $\mathrm{SD}=.86$ & & & \\
Treatment Method & $\mathrm{M}=1.9$ & $\mathrm{M}=2.03$ & $(190) .874$ & .380 & .13 \\
\hline
\end{tabular}

Correlational analysis of the dependent variables (structural principle and optimal treatment method) and covariates (prior experience and demographics) did not reveal any significant relationships $(p=>.05)$. Therefore, based on these findings, the covariates were assessed not to have had an impact on transfer outcomes, negating the need for ANCOVA analysis.

\section{DISCUSSION}

Historically, students pursuing an allied health degree have received the majority of their professional education and training based on the single case method of instruction. ${ }^{20}$ As a result of shortcomings of this method in promoting transfer of learning, greater attention is being paid to documenting the benefits of using multiple case studies, particularly among novice learners. ${ }^{5}$ Outcomes of our study, which are consistent with those in the cognitive sciences literature, suggest multiple case examination with cueing to be a more effective pedagogical technique over multiple case examination alone to improve learning transfer and problem solving.,111,18,21 Additionally, our findings support the underpinnings of case-based analogical reasoning with cueing for novice health care students, suggesting that cueing students to look for structural relationships across multiple patient cases assists in 1) identification of the cases shared structural principle(s), (b) builds schema that result in more effective problemsolving and 2) fosters learning transfer.

Seel has argued though that solution of complex real-world problems may not be teachable at all through a mock analogical reasoning process. ${ }^{14} \mathrm{He}$ contended problems must be "experienced and dealt with using general intelligence and world knowledge".14,p47 Moreover, critics may also point out that novices not only need similar prior experience to engage in successful analogical reasoning and transfer, but must also possess proficiency in the respective domain: albeit; without experience, no opportunity exists even to develop proficiency, let alone recall the experience for application. However, Gentner et al showed that prior experience with a source analogue or domain-although helpful to engender analogical reasoning-is not absolutely necessary because multiple case examination with cueing supplants lack of prior experience within the domain and serves to fill relational gaps in individuals' experience, thereby providing them a foundation upon which to compare and solve novel problems. ${ }^{4}$

Our findings for transfer of the structural principle affirm the value of using a case-based analogical reasoning process with cueing to improve transfer in a pre-professional undergraduate novice population. Cued participants who demonstrated greater transfer of the structural principle likely did so because they were prompted to actively look for and compare the structural similarities between the heat-illness cases, unlike non-cued participants who analyzed the cases separately. Moreover, cued participants may have formed a perception from the cues that comparison of the cases would help them solve a future problem, therefore improving transfer of the structural principle. Speicher and Kehrahn in their Perception of Applicability Model posit that it is essential for the learner to form a perception that their learning experience is applicable in order for learning transfer to occur. ${ }^{12}$

As stated previously, our study was designed to expand on the work of Norman et al who found a very large effect size difference $(d=1.36)$ in solving clinical case problems between undergraduate health sciences students who examined multiple case examples and received cues and students who just utilized multiple cases. ${ }^{5}$ Though we also identified a significant difference in learning transfer in favor of cued participants, the effect size in our study was noticeably smaller $(d=.39)$. The lack of robustness in effect between groups may have been due to a possible limitation in how the cueing intervention was delivered.

In order to control variance in our study, we chose to deliver the cueing intervention through written instruction instead of guided verbal instruction, which has been shown to be effective in promoting transfer. ${ }^{4}$ Gentner et al found undergraduate business students who received guided instruction (probing participants with questions related to their understanding of comparison cases 
for application to a test case) proposed more solutions and demonstrated better transfer of a business negotiation principle to solve a business problem than participants who received simple or no instruction. ${ }^{4}$ These results point to the value of providing students with real-time guidance and feedback to assist them in calibration of their thinking when engaged in a case-based analogical reasoning process. Even though in our study, paper-based cueing ensured more consistent delivery of cases and their cues, the format did not provide learners' feedback on how the cases or the structural principle(s) might be interpreted or applied. The paper-based delivery format may have also caused cognitive overload of the participants. ${ }^{22}$

When the capacity of working memory is limited or exceeded, cognitive overload occurs.22 The inability of our participants to reference the worked-cases and respective analyses when attempting to solve the target case may have unduly taxed their working memory, causing cognitive overload that inhibited transfer and problem solving. Cognitive load theory suggests that individuals have a limited short-term working memory capacity that can be overwhelmed with complex task requirements.22,23 We believe that even though our participants were provided worked case examples with solutions and ample time to complete the problems, the lack of verbal explanation coupled with the need to remember the cases and analyses when attempting to solve the third case may have demanded a greater amount of working memory to process the structural attributes of the cases, thwarting a more positive transfer effect.

With regard to the second dependent variable, transfer of the optimal treatment method to the target case, the cueing intervention did not have a significant impact. A simple explanation may exist for this finding. Novick found when novices attempt to solve a problem, they are likely to retrieve similar past experiences to form their solution procedure even when cued to use a correct solution procedure that has been provided. ${ }^{24}$ Lacking a significant difference in our groups based on prior experience with the case content, the likelihood exists that the lack of transfer of the optimal treatment method was the result of either how the cues or cases were constructed; cued participants may not have recognized the future utility of the optimal treatment method utilized in the heat-illness cases. That is, the written cue or solution of the hypothermic case may have been so simple and intuitive in nature (i.e., apply heat directly to the patient) that the cueing intervention had a negligible influence on transfer of the treatment method.

In sum, keeping in mind the limitations in the present study, cued participants transferred the structural principle more than noncued participants and solved the dissimilar patient case more effectively. We caution readers in generalizing the study and its outcomes to other teaching methods (e.g., problem-based learning, web-based learning, etc.) and non-academic settings (e.g., workplace training) because the study setting occurred in a traditional undergraduate academic classroom environment. Clinically, however, the study implications are significant.

\section{IMPLICATIONS}

In 2001, the Institute of Medicine (IOM), a committee of physicians and health policy experts charged to improve the health of the nation by the U.S. National Academy of Sciences, identified a gap in the area of education of health care practitioners as one of the plausible reasons for medical error. ${ }^{25}$ We along with other researchers believe that traditional instructional strategies such as single case examination are insufficient to enable students to transfer what they have learned in the classroom to address novel clinical problems they will face as future health care professionals. $3,5,18,27-29$ The challenge for educators of health care students and adult learning practitioners is to construct and facilitate learning experiences that capitalize on identification of structurally relevant case comparison examples for solution of future clinical problems.

\section{CONCLUSION}

The findings of our study are a first step towards addressing a small but critical facet of the medical error phenomenon and overall lack of transferability of classroom instruction to clinical practice. Two observations from our findings are particularly relevant based on the context of educating future health care practitioners. First, learning transfer and problem solving are improved with the use of a paper-based format that provides pre-professional health profession students cues when examining multiple worked cases, a potentially useful pedagogical tool to engender learning for novice students in large classes. Second, a paper-based format may not be as useful for teaching treatment approaches unless constructed in a manner that allows for depth in the application of shared principle(s) or concept(s) and for guidance and feedback in the process. Future research should explore how the pedagogical approach utilized in this study works over an extended period of time with allied health students, medical students, and practicing clinicians. Additionally, studies should be conducted to determine what role and magnitude a perception of applicability of a case-based analogical reasoning learning experience plays in the motivation of individuals to transfer classroom learning to clinical practice. 


\section{REFERENCES}

1. Barnett SM, Ceci SJ. When and where do we apply what we learn? A taxonomy for far transfer. Psychol Bull. 2002; 128(4):612-37. [PMID12081085]

2. Radtke S. A conceptual framework for clinical education in athletic training. Athl Train Educ J. 2008;2:36-42.

3. Shine KI. AAMC Paper. Acad Med. 2002;77:91-9. [PMID 11788332]

4. Gentner D, Loewenstein J, Thompson L. Learning and transfer: a general role for analogical encoding. J of Educ Psychol. 2003;95(2):393-408.

5. Norman G, Dore K, Krebs J, Neville AJ. The power of the plural: effect of conceptual analogies on successful transfer. Acad Med. 2007;82(10):S16-8. [PMID 17895680]

6. Gentner D, Smith L. Analogical reasoning. In V. S. Ramachandran (Ed.) Encyclopedia of Human Behavior (2nd Ed.). Oxford, UK: Elsevier; 2002:130-6.

7. Speicher TE, Bell A, Kehrhahn M, Casa DJ. Case-based analogical reasoning: a pedagogical tool for promotion of clinical reasoning. Athl Train Educ J. 2012;7(3):129-36.

8. Gentner D. Structure-mapping: a theoretical framework for analogy. Cogn Sci. 1983;7(2):155-70.

9. Speicher TE. Effect of cueing on learning transfer among pre-professional undergraduate healthcare students engaged in a case-based analogical reasoning exercise. Paper presented at: Adult Education Research Conference \& the Canadian Association for the Study of Adult Education Annual Meeting; June 11, 2011; Toronto, Ontario Canada.

10. Holyoak KJ, Koh K. Surface and structural similarity in analogical transfer. Mem Cogn. 1987;15(4):332-40. [PMID 3670053]

11. Shayo C, Olfman L. The role of training in preparing end users to learn related software. J End User Comput. 2000;12(1):313.

12. Speicher TE, Kehrhahn M. Analogical reasoning: a process for fostering transfer of learning from the classroom to the clinical practice setting. Int Forum Teach Stud. 2009;5(2):52-8.

13. Chi MT, Feltovich PJ, Glaser R. Categorization and representation of physics problems by experts and novices. Cogn Sci. 1981;5(2):121-52.

14. Seel NM. Mental models and complex problem solving: instructional effects. In: Handling Complexity in Learning Environments: Theory and Research. Philadelphia: Elsevier; 2006:43-66.

15. Duncker K, Lees L. On problem-solving. Psychol Monogr. 1945;58(5):1-113.

16. Creswell JW. Educational research: Planning, conducting, and evaluating quantitative and qualitative research (4th ed.). Upper Saddle River, NJ: Pearson Education; 2012.

17. Gick ML, Holyoak KJ. Schema induction and analogical transfer. Cogn Psychol. 1983;15(1):1-38.

18. Gentner D, Colhoun J. Analogical processes in human thinking and learning. In: von Müller A, Pöppel E, Glatzeder B, Goel V, von Müller A, eds. Towards A Theory on Thinking: Building Blocks for a Conceptual Framework. London: Springer; 2010:35-49.

19. Cohen J. Statistical power analysis for the behavioral sciences (2nd ed.). New Jersey: Lawerence Erlbaum Associates; 1988.

20. Sage WM. Medical liability and patient safety. Health Affairs. 2003;22(4):26. [PMID 12889746]

21. Novick LR, Holyoak KJ. Mathematical problem solving by analogy. J Exp Psychol Learn. 1991;17(3):398-415. [PMID 1829473]

22. Tuovinen JE, Sweller J. A comparison of cognitive load associated with discovery learning and worked examples. J Educ Psychol. 1999;91:334-41.

23. Kirschner PA, Sweller JE, Clark RE. Why minimal guidance during instruction does not work: an analysis of the failure of constructivist, discovery, problem-based, experiential, and inquiry-based teaching. Educ Psychol. 2006;41(2):75-86.

24. Novick LR. Analogical transfer, problem similarity, and expertise. J Exp Psychol Learn. 1988;14(3):510-20. [PMID 2969945]

25. Committee on Quality Health Care in America, IOM. Crossing the quality chasm: a new health system for the 21 st century. Washington, DC: National Academy Press; 2001.

26. Clark R, Harrelson GL. Designing instruction that supports cognitive learning processes. J Athl Train. 2002;37(4):S152-9. [PMID 12937537]

27. Hummel HGK, Nadolski RJ. Cueing for schema construction: designing problem-solving multimedia practicals. Contemp Educ Psychol. 2002;27(2):229-49.

28. Weeks KW, Lyne P, Mosely L, Torrance C. The strive for clinical effectiveness in medication dosage calculation problemsolving skills: the role of constructivist learning theory in the design of a computer-based 'authentic world' learning environment. Clinical Effectiveness in Nursing. 2001;5(1):18-25.

29. Weeks KW, Lyne P, Torrance C. Written drug dosage errors made by students: the threat to clinical effectiveness and the need for a new approach. Clinical Effectiveness in Nursing. 2000;4(1):20-9. 
APPENDIX A

Demographic and Prior Experience Survey

Demographic and Prior Experience Survey (DPES)

Demographic Information

Please answer the following questions:

How old are you?

What is your gender?

What geographic location have you spent the most of your life?
$€$ Northeast
$€$ Southeast
$€$ Midwest
$€$ Northwest
$€$ Southwest
$€$ Other:

What is your Race? Select one or more of the categories listed:

$€$ American Indian or Alaska Native

$€$ Asian

$€$ Black or African American

$€$ Native Hawaiian or Other Pacific Islander

$€$ White

$€$ Some Other Race:

Prior Experience Information

Circle either Yes or No for each question:

Yes / No Have you ever had a heat illness (e.g., heat exhaustion, heat stroke)?

Yes / No Have you ever had a cold illness (e.g., hypothermia, frostbite)?

Yes / No Have you ever received formal instruction on heat or cold illnesses?

Yes / No Have you ever treated an individual with a heat or cold illness?

Yes / No Have you ever observed an individual who has experienced a heat or cold illness?

Yes / No Have you ever read any materials about heat or cold illnesses? 
APPENDIX B

Learning Transfer Assessment Instrument

Learning Transfer Assessment Instrument (LTAI)

Rate separately, the amount of structural similarity of the solution principle and warming method provided by the subject for the target case to that of the inherent optimal solution principle in the base cases, which is thermoregulation of core body temperature through application of a direct full body heating technique.

Amount of Structural Similarity Present in Target Case Solution

$0 \quad$ Not at all

$1 \quad$ To a small extent

2

To some extent

3 To a great extent
Example: The individual should be rushed to the hospital.

Thermoregulation principle and heating technique is NOT mentioned

Example: They need to get warm again by getting out of the whirlpool.

Thermoregulation principle present, but vague and patient centered

Heating technique is passive and patient centered

Example: Warm the body by placing blankets over the body.

Thermoregulation principle present, vague, but clinician centered

Heating technique is direct but does is NOT applied full body

Example: Raise body temperature by wrapping individual with warm blankets.

Thermoregulation principle is explicitly present and is clinician centered

Heating technique is direct and applied to the full body and also involves a

heating source (e.g., warm blankets) 